\title{
Diagnose und Therapie
}

P. G. Livingstone and H. M. Walker, A Study of the Effects of Liquid Mustard Gas upon the Eyes of Rabbits and of certain Methods of Treatment. British Journ. of Ophthalm. 24, 67, 1940. Es ist wohlbekannt, daß das Gelbkreuzgas auf die lebenden Gewebe einen gewaltigen chemischen Reiz ausübt, der nicht allein im ersten Augen-blick einwirkt, sondern länger fortdauert du $\mathrm{Cch}$ seine sehr toxischen Bei-produkte. Verf. haben Versuche angestellt liber verschiedene Methoden, um die Augenverãnderungen, durch dieses Kampfgas verursacht, zu verhindern oder zu lindern. Bei Kaninchen wurde der kleinstmögliche Tropfen (max. Größe 0,75 $\mathrm{cmm}$ ) aus einer Glaspipette zwischen 11 und $1 \mathrm{Uhr}$, ungefähr $2 \mathrm{~mm}$ vom Limbus entfernt, auf die Hornhautoberfläche appliziert. Das Auge wurde dann eine Minute offen gelassen, während das dritte Lid durch einen Lidhalter zurückgezogen blieb, so daß die Wirkung bei alien Augen ungefähr dieselbe war.

Die benutzten Lösungen und Stoffe zum Ausspülen und Eintropfen des Bindehautsackes waren die folgenden. Zum Ausspülen wurde (nach einer Anzahl von Vorversuchen) benutzt Bleichpulver 1:400 (pH 10,0), Lösung von doppeltkohlensaurem Natrium 2\% (pH 8,0), Kochsalzlösung von 1,4\% (pH 6,8), Bonnefons Lösung, d. i. eine gesättigte Lösung von $800 \mathrm{~g}$ Natrium-sulphat mit $200 \mathrm{~g}$ Zuckerlösung (pH 7,0). Zum Eintropfen wurde benutzt: Lebertran mit Bleichpulver, flüssiges Paraffin mit Bleichpulver, Benzoyl-peroxyd, ferner Oel Nr. 1 (flüssiges Paraffin mit Vitamin A 12000 Ein-heiten), Oel Nr. 2 (Arachidöl), Oel Nr. 3 (Lebertran mit Vitamin A 12000 Einheiten und Vitamin D 2000 Einheiten).

Die Beginnausspülungen (d. h. solche, die ganz kurz nach dem Eintropfen der Gelbkreuzlösung vorgenommen wurden) dauerten 5 Minuten, während spätere Ausspülungen (also solche während der Behandlung) $3 / 8$ Minute dauerten. Beim Eintropfen der übrigen Substanzen wurden jedesmal 8 Tropfen gegeben. Von der Merthiolat-Lösung für antiseptische Zwecke wurde bei jeder Behandlung $4 \mathrm{ccm}$ gegeben nach vorheriger gründlicher Reinigung mit Wasser.

Bei Vorversuchen waren die Verf. auf mehrere wichtige Punkte ge-stoßen. In erster Reihe zeigte es sich, daß man eine Beginnperiode der Ein-wirkung der Gelbkreuzflüssigkeit und eine zweite der Ausbreitung unter-scheiden muß. Bei der zweiten entstehen tiefe Hornhaulveränderungen, und diese können durch eine Oberflächenbehandlung nicht mehr beeinflußt wer-den. Man muß das zweite Stadium, das der Verbreitung in die Tiefe, also zu verhindern suchen, und zwar durch Steigerung des natürlichen Wider-standes der Gewebe, was nur durch den Blutstrom geschehen kann. Die fortschreitenden Gewebsänderungen der Hornhaut werden wahrscheinlich nicht durch das Gelbkreuzgas als solches verursacht, sondern durch Bindung an Aminogruppen des Eiweißes, wodurch feste toxische Substanzen entstehen. Als Substanz, die möglicherweise die natürlichen Widerstandskräfte der Cornea verstärken kann, wurde Ascorbinsäure (500 mg intravenös ein-gespritzt) versucht.

Klinische Beobachtung der Gelbkreuzvergiftung. Die direkte örtliche und sehr schnelle Wirkung des Gelbkreuzgases wird

254

Diagnose und Therapie 
bemerkbar duГch die Vemichtung des Hornhautepithels mit Einschluß der Bowmanschen Membran, so daß starke Färbuutg durch Fluorescin der Horn-haut auftГitt. Diese Ae $\pi$ derungen treten sowohl bei unbehandelten Fallen auf als bei solchen, die während fünf Minuten ausgespült waren. Nach 24 Stun-den hat sich das Bild sel· $\Pi$ - geändert. Die färbbare Zone braucht zwar nicht größer geworden zu sein, abe $\Gamma$ es besteht nun ein starkes Hornhautödem mit Abscheidung einer mehr oder weniger dicken weißen Substanz und Blasen-bildung der Bindehautfalten. In einem Drittel der Fälle ist die Iris zusam-mengezogen. Nach 48 Stunden kann man gewöhnlich durch die tiefe Keratitis die Iris nicht mehr gut sehen, und nach vier Tagen ist die Absonderung gewöhnlich so stark, daß die Lider verklebt sind. Die Färbung der Hornhaut ist in diesem Stadium geringer geworden, die Iris hat ihre gewöhnliche Form wieder erreicht. Die Lidschädigung wird nun sehr bemerkbar: Ectropion und Membranbildung, gefolgt von Narbenbildung, treten bisweilen auf.

Bei weitern Experimenten wurde das Resultat der Einspritzung von Gelbkreuzöl in den Bindehautsack studiert, nachdem fünf oder fünfzehn Minuten nach der Einverleibung des Tropfens eine Auswaschung vorgenom-men war. Nach sieben Tagen wurde die heilende Wirkung von Merthiolat 1:10 000 und Lebertran versucht. Das Resultat dieser Behandlung konnte man sehr zufriedenstellend nennen.

Betrachtet man nun die Behandlung, so ist es nötig, drei Stadien der Einwirkung des Gelbkreuzöls ins Auge zu fassen.

Das Stadium der direkten fatalen Einwirkung, wobei nicht allein die oberflächlichen Hornhautepithelien getötet werden, sondern auch tiefer liegende Zellen, wonach das Gift weiter durchdringt.

Das Stadium der Weiterverbreitung. Die Behandlung zielt darauf hin, die sekundäre weiterschreitende Keratitis aufzuhalten. Dieser Infiltration wird entgegengearbeitet:

durch den natürlichen Widerstand der Gewebe, der bei den einzelnen Personen sehr verschieden zu sein scheint;

durch Lebertran, das irgendeine Substanz zu enthalten scheint, die sowohl schützend wie heilend wirkt.

Das Stadium der se $1 / 8$ ndären Infektion. Es wurden in den Ausstrichen nur wenig Mikroorganismen gefunden. Dagegen kamen massive Desqua-mationen viel mehr vor. Merthiolat zeigte sich sehr geeignet zur Heilung dieses Zustandes, besonders unter Zufügung von Lebertran. Es zeigte sich ferner, daß ein Vorteil dadurch erreicht wird, daß Lösungen eng mit den Geweben in Kontakt treten. Besonders ist das da nötig, wo Oedem und Blepharospasmus besteht. Ein Irrigationsspeculum ist zur Ausspülung empfehlenswert. Die Auswirkung von Askorbinsciure wurde an einer kleinen Gruppe von Kaninchen studiert. Diese bekamen während sechs Tagen täglich $500 \mathrm{mg}$ C-Vitamin eingespritzt. Fünf Minuten nach Ein-bringung des Gelbkreuzgiftes wurde das rechte Auge der Kaninchen mit 2\% doppeltkohlensaurem Natrium und Lebertrantropfen behandelt.

Im allgemeinen waren diese rechten Augen nach geringen Entzündungserscheinungen nach etwa sieben Tagen wieder geheilt. Bei den linken, nicht behandelten Augen beslanden die Entzündungserscheinungen längere Zeit, besonders feine comeale Trübung und leichte

Narbenbildung des obern und

Diagnose und Therapie

255 
untern Lides. Am elften Tage war der Zustand auch am linken Auge abe $\Gamma$ fast wieder normal. $\mathrm{Ab}$ und zu wurde Pigment in den Hornhautwunden nachgewiesen, was wahrscheinlich vom innern Rande des dritten Lides herrührte.

Die Schlußfolgerung, die man aus dieser letzten Versuchsreihe ziehen kann, ist, daß Askorbinsåure offenbar ein starkes Vorbeugungsmittel bildet gegen den gewöhnlich verheerenden Einfluß von Gelbkreuzgas auf das Horn-hautgewebe.

Die histologischen Verãnderungen sind am stärksten im Epithel, weni-ger stark in den tiefer gelegenen Geweben.

Schwellung der Epithelzellen, Chromatolyse ihrer Kerne, deutlichere Zellgrenzen, Desquamation der oberen Zellschichten bilden das allgemeine Bild.

Im Stroma sind die Zellen geschwollen, so daß der in Mitleidenschaft gezogene Teil der Hornhaut dicker ist als der übrige Teil. Später findet Kern-vermehrung statt, und es wandern pseudoeosinophile Zellen in das Stroma. Diese Zellen kommen aus den verdickten Gefäßen am Limbus zum Vorschein. An der Descemet werden keine bestimmten Aenderungen wahrgenommen, aber in schweren Fallen war das Endothel geschwollen und teilweise desquamiert. Auch kann man bisweilen in solchen schweren Fallen ein albuminöses Ex-sudat in der Vorderkammer wahrnehmen, das aber meistens später ver-schwindet.

Schließlich beschreiben die Verfasser einen Lidhalter mit drei Armen, wodurch Spülung des Bindehautsackes und der Hornhaut bequemer wird. Für das menschliche Auge glauben sie aber nicht, daß eine Hornhautver-schwürung durch solche Lidhalter verhindert werden kann.

E.Marx $\uparrow$ (Rotterdam).

Olivier de Saint-Martin: Contribution à $\Gamma$ Etude des Anesthésies post opératoires dc la Cornée. These. Toulouse. 1939.

Der Verfasser hat an 74 Starkranken mit den v. Freyschen Reizhaaren nach der Technik von v. Frey und Marx die nach Anlegung des Halbbogen-schnittes nach oben mit dem Groe/e-Messer veränderte Sensibilität der Hornhaut geprüí't. 1. An 4 Fallen während des Operationsmonates, 2. an 60 Fallen nach 1 Monat und im Laufe des Operationsjahres, 3. an 6 Fallen später als Jahr nach der Operation. Um die empfindlichen Augen nicht allzusehr zu reizen, wurde die Zahl der Testpunkte verkleinert. Die Hornhaut wurde in je

halbmondförmige Sektoren nach oben und unten von je $2 \mathrm{~mm}$ Höhe ein-geteilt, so daß ein ovaler Bezirk von $3 \mathrm{~mm}$ Höhe in der Mitte verblieb. In jedem Sektor wurden 4 Punkte geprüft, im ganzen je Auge 20 Berührungen. Die Pruning begann mit biegsamen Reizhaaren von $5 \mathrm{mg}$ und stieg bei negativem Befund zu immer härteren Haaren bis zu einem Höchstgewicht von $300 \mathrm{mg}$ $=$ völlige Gefühllosigkeit. Physiologisch fand er die Schwelle der Gefühlsempfindung mit steigenden Jahren herabgesetzt, und zwar im Alter von 60 Jahren und darüber in 29,72\% seiner Fälle. Bei Gesunden waren die mittleren Werte 5-10 mg, nicht wie in den Befunden von Krückmann X-2 mg. In 22 Fallen wurden 20-30 mg, einmal 50 und bei einem Diabe-tiker 100 mg festgestellt.

256 Buchbesprechungen - Books Review - Livres Nouveaux

Die Untersuchungsbefunde sind in übersichtlichen, schematischen Zeich-nungen topographisch, nach der fur die Wiederherstellung der Gefühls-empfindung notwendigen Zeit, dem Alter des Operierten sowie nach son-stigen allgemeinen und lokalen Gesichtspunkten ausgewertet. Die Ergebnisse sind folgende:

Jeder Starschnitt in der oberen Limbushälfte mit dem Grae/e-Messer verursacht eine Gefühllosigkeit der Hornhaut, die in den ersten Wochen nach dem Eingriff fast immer die ganze 
Hornhaut betrifft und ohne Aus-nahme die Grenzen des Lappens unterhalb des horizontalen Meridians über-schreitet.

Die Wiederherstellung der Gefühlsempfindung geschieht in der großen Mehrzahl der Fälle von unten nach oben, bzw. vom Zentrum zur Peripherie. Die Gefühllosigkeit, bzw. Verminderung kann Monate, evtl. Jahre andauern, besonders im oberen Sektor unterhalb der Narbe, wo die Gefühlsverminde-rung an sich stets am stärks $1 / 8 \mathrm{n}$ ist.

Infolgedessen ergeben sich drei Hauptformen in der topographischen Wiederherstellung der Gefühlsempfindung: a) nur im unteren limbusnahen Sektor; b) drei Zonen abnehmender Gefühlsempfindung von unten nach oben; c) Gefühlsempfindung im unteren Sektor wiederhergestellt, im oberen noch aufgehoben, bzw. erheblich vermindert.

Die Intensität der Gefühlsverminderung richtet sich nach der Zeit, die seit der Operation verstrichen ist, dem Alter des Patienten, seinem Allge-meinzustand, dem Operations- und Heilverlauf.

Außer in den Fallen mit Komplikationen und anormaler Narben-bildung konnte an keinem Auge mit Gefühlsverminderung oder Gefühllosigkeit an der Spaltlampe ein pathologischer Befund erhoben werden,

Es ist zu empfehlen, daß sich Operierte mit Gefühlsstörungen der Hornhaut, ganz besonders solche mit Gefühllosigkeit, noch Monate nach derOperation möglichst vorsichtig verhalten. Heßberg, z. Zt. Lausanne.

Buchbesprechungen - Books Review - Livres Nouveaux

G. von Studnitz: Physiologie des Sehens. Retinale Primärprozesse. 367 S.,

60 Abb. Akademische Verlagsgesellschaft m. b. H., Leipzig, 1940. Broschiert

RM. 22.-, gebunden RM. 24.-.

I. In der Reihe der Monographien «Probleme der Biologie» erscheint als drifter Band das vorliegende Buch. Es versucht zum erstenmal eine zusam-menfassende Darstellung der bisher bekannten objektiven Vorgänge im peri-pheren Organ in ihren Beziehungen zu den physiologisch-optischen Phäno-menen. Insbesondere wird die Dunkeladaptation berücksichtigt. Verfasser der selbst auf diesem Gebiete wichtige Beiträge geleistet hat u. a. die Entdeckung eines Sehstoffes in den Zapfen während der Sehpurpur in den Stäbchen ja schon lange bekannt ist versteht es die verwickelten Vorgänge in dem peri-pheren Endorgan (retinale Primärprozesse) darzustellen auch für denjenigen der mit den einschlägigen Problemen nicht so nahe vertraut ist. Für den Fachmann ist namentlich durch die ausgiebige Berücksichtigung der Lite-ratur das Werk zur schnellen Orientierung über spezielle Fragen wichtig.

Bruckner. 